\title{
Constrained fluctuation theories of rubber elasticity: General results and an exactly solvable model
}

\author{
R. Everaers ${ }^{\mathrm{a}}$ \\ Institut Charles Sadron, 6 rue Boussingault, 67083 Strasbourg Cedex, France \\ and \\ Max-Planck-Institut für Polymerforschung, Postfach 3148, 55021 Mainz, Germany
}

Received: 28 November 1997 / Received in final form and accepted: 31 March 1998

\begin{abstract}
We present a new model of rubber elasticity where linear forces act to constrain the fluctuations of the eigenmodes of the phantom model. The model allows us to treat the constrained junction and the tube model within the same, transparent formalism, does not require any further approximations, and is particularly suited for the analysis of simulation data for (strained) model polymer networks. As an interesting side result we show that in order for the model to be consistent, the constraints (but not the mean polymer conformations!) have to deform affinely, a severe restriction that might also apply to other models. Complementary, we prove in analogy to the derivation of the virial theorem that introducing constraints into the phantom network Hamiltonian leads to extra terms in addition to the usual Doi-Edwards formulas for the polymer contribution to the stress tensor which vanish only for affinely deforming constraints.
\end{abstract}

PACS. 05.40. $+\mathrm{j}$ Fluctuation phenomena, random processes, and Brownian motion - 62.20.Dc Elasticity, elastic constants - 61.41.+e Polymers, elastomers, and plastics

\section{Introduction}

Most current models of rubber elasticity are based on the phantom model [1-3] combined with the idea that entanglements between the polymer chains reduce the fluctuations [4-21]. Being deformation dependent, the effective constraints then contribute to the elastic properties of the network. What divides the community, however, is the question which fluctuations are affected by the mutual impenetrability of the network strands.

The classical theories [1-9] date back more than half a century and can qualitatively explain many aspects of the physics of rubber elasticity. They are based on the observation that a flexible, randomly coiled polymer in a melt with a mean-square end-to-end distance $\left\langle r^{2}\right\rangle$ can be viewed as a linear entropic spring with spring constant $k=\frac{3 k_{B} T}{\left\langle r^{2}\right\rangle}$ and assume that the elastic response of rubber has its sole origin in the elongation of the network strands. In the two original classical models, the phantom model and the junction affine model, the junction points are considered to be free to fluctuate or fixed in space respectively. Later refinements considered partially restricted fluctuations.

Edwards [10] argued in 1967 that the average effect of the complicated topological constraints on the conforma-

\footnotetext{
a Present address: Institut Curie, Section de recherche, 11 rue Pierre et Marie Curie, 75231 Paris Cedex 05, France.

e-mail: Ralf.Everaers@curie.fr
}

tion of a network strand is to confine the strand to the neighborhood of a mean conformation. The tube model provides on a mean-field level a unified view on networks and entangled polymer melts [11-14]. There is convincing evidence from simulations [22-25] as well as from neutron scattering experiments [26-30] for the existence of the tube, but it still remains to be shown that it is possible to calculate the elastic response from this ansatz.

That the importance of entanglements is still sometimes disputed is due to the inconclusiveness of rheological experiments [31-37]. It is very complicated to prepare well-characterized model systems and to relate experimental data to structural properties or to analytical theory in a unique way. Due to the lack of microscopic information theories can only be tested indirectly by comparing measured and predicted stress-strain curves or by extrapolating the moduli to the limit of vanishing cross-link density. This has lead to ambiguous and contradictory results. Although SANS investigations of deformed networks have partially closed this gap $[29,30]$, we have argued [38], that due to the direct and simultaneous accessibility of microscopic and macroscopic information, computer simulations (for a recent review see Ref. [39]) are in a much better position to decide these issues. In the ideal case, it should be possible to test statistical mechanical models of rubber elasticity without using any adjustable parameters. Two questions are of principal interest:

1. How do the microscopic chain conformations change in response to a macroscopic deformation? 
2. What is the relation between the (change of the) microscopic conformations and macroscopically observed stresses and deformation free energies?

Theories worked out for a comparison to experimental data are not necessarily well-suited for a quantitative test of the underlying ideas. For example, many theories describe limiting cases such as networks with strand lengths much smaller or larger than the melt entanglement length, as it is the case for the constrained junction and tube models respectively. Simulations and experiments, on the other hand, often fall into the crossover region between these two extremes. The purpose of the present paper is the introduction of a conceptually transparent framework for the analysis of constrained fluctuations and their contribution to the shear elastic properties of a polymer network and to illustrate some subtleties concerning the second question related to the presence of constraints.

The paper is organized as follows. First we review the phantom model which treats the network strands as ideal entropic springs and completely ignores entanglements. In the phantom model, the eigenmodes only depend on the connectivity and not on the size and shape of the network and therefore do not contribute to the shear modulus. In Section 3 we introduce the constrained mode model (CMM) where linear forces act to constrain the fluctuations of the eigenmodes of the phantom model. The model is exactly solvable and makes no assumptions on the length of the network strands. In the discussion (for details see the appendix) in Section 4 we point out how the constrained junction and the tube model can be obtained as simple limiting cases of the CMM. We close by some general considerations concerning the deformation dependence of the constraints. Quite surprisingly, one is within the CMM almost restricted to the simplest possible choice, affinely deforming constraints. In particular, most of the functional forms which were used in other constrained flucutation models of rubber elasticity in order to explain non-affine chain deformations and to improve the agreement with measured stress-strain curves lead to inconsistencies in the present case. While we have no general arguments against the use of non-affinely deforming constraints, we show that they generate extra terms in the stress tensor in addition to the usual Doi-Edwards expression. A quantitative test of the CMM and of the DoiEdwards formula in computer simulations of model polymer networks will be presented in a future publication [40].

\section{The phantom model}

The Hamiltonian of the phantom model [1-3] is given by

$$
\mathcal{H}_{p h}=\frac{k}{2} \sum_{\langle i, j<i\rangle} r_{i j}^{2},
$$

where $\langle i, j<i\rangle$ denotes a pair of nodes which are connected by a polymer chain and $\mathbf{r}_{i j}(t)=\mathbf{r}_{i}(t)-\mathbf{r}_{j}(t)$ the distance between them. Due to the linearity of the springs the problem separates in Cartesian co-ordinates $x, y, z$.
Furthermore, a conformation of a network of harmonic springs can be analyzed in terms of either the bead positions $\mathbf{r}_{i}(t)$ or the deviations $\mathbf{u}_{i}(t)$ of the nodes from their equilibrium positions ${ }^{1} \mathbf{R}_{i}$. The latter are characterized by a force equilibrium $\sum_{j} \mathbf{R}_{i j} \equiv 0$, where $j$ indexes all nodes which are connected with node $i$. In this representation, the Hamiltonian separates into two independent contributions from the equilibrium extensions of the springs and the fluctuations, where the latter can be written as a sum over independent normal modes or phonons $\mathbf{u}_{p}[41,42]$ :

$$
\begin{aligned}
\mathcal{H}_{p h} & =\frac{k}{2} \sum_{\langle i, j<i\rangle} \mathbf{R}_{i j}{ }^{2}+\frac{k}{2} \sum_{\langle i, j<i\rangle} \mathbf{u}_{i j}{ }^{2} \\
& =\frac{k}{2} \sum_{\langle i, j<i\rangle} \mathbf{R}_{i j}{ }^{2}+\frac{k_{p}}{2} \sum_{p} \mathbf{u}_{p}{ }^{2} .
\end{aligned}
$$

In the second form, the calculation of the shear modulus of the phantom model from the deformation dependence of the free energy is formally straightforward:

$$
\begin{aligned}
F_{p h}(\lambda)= & \frac{k}{2} \sum_{\langle i, j<i\rangle} \mathbf{R}_{i j}{ }^{2}(\lambda) \\
& -k_{B} T \sum_{p} \log \left(\int d^{3} u_{p} e^{-\frac{1}{k_{B} T} \frac{k_{p}}{2} \mathbf{u}_{p}{ }^{2}}\right) .
\end{aligned}
$$

\section{Elastic properties}

In this paper we always consider uni-axial elongations

$$
\overleftrightarrow{\lambda}=\left(\begin{array}{lll}
\lambda & & \\
& 1 / \sqrt{\lambda} & \\
& & 1 / \sqrt{\lambda}
\end{array}\right)
$$

This volume-conserving deformation $(\operatorname{det}(\overleftrightarrow{\lambda})=1)$ is the standard choice [4] for rubber-like materials, since they can be considered to be incompressible with a Poisson ratio of $\frac{1}{2}$. The corresponding Lagrangian strain tensor is

$$
\overleftrightarrow{\epsilon}=\frac{1}{2}\left(\overleftrightarrow{\lambda}^{t} \overleftrightarrow{\lambda}-1\right)
$$

where the superscript ${ }^{t}$ indicates matrix transpose. The deformation dependent free energy and the shear modulus

\footnotetext{
${ }^{1}$ Formally, a phantom network collapses to the size of one network strand when the Hamiltonian (1) is used with open boundary conditions. In reality, this collapse is prohibited by volume interactions between the chains. Within the phantom network model one can avoid the collapse by either fixing some junction points on the sample surface $[1,2]$, or, more conveniently, by spanning the network over a fixed volume with the help of periodic boundary conditions. See R.T. Deam, S.F. Edwards, Phil. Trans. R. Soc. A 280, 317 (1976).
} 
of the phantom model are then given by

$$
\begin{aligned}
F_{p h}(\lambda) & =E_{0}(\lambda)+\frac{3 k_{B} T}{2} \sum_{p} \log \left(\frac{k_{p}}{k_{B} T}\right) \\
E_{0}(\lambda) & =\frac{k}{2} \sum_{\langle i, j<i\rangle}\left(\overleftrightarrow{\lambda} \mathbf{R}_{i j}\right)^{2} \\
G_{p h} & =\left.\frac{1}{3} \frac{1}{V} \frac{d^{2} F_{p h}}{d \lambda^{2}}\right|_{\lambda=1}=\left.\frac{1}{3} \frac{1}{V} \frac{d^{2} E_{0}}{d \lambda^{2}}\right|_{\lambda=1} \\
& =\frac{\left\langle R_{\text {strand }}^{2}\right\rangle}{\left\langle r^{2}\right\rangle} \rho_{\text {strand }} k_{B} T
\end{aligned}
$$

where $\left\langle r^{2}\right\rangle$ the mean square end-to-end distance of the un-cross-linked strands in a melt, and $\left\langle R_{\text {strand }}^{2}\right\rangle=\left\langle R_{i j}^{2}\right\rangle$ the expectation value of the square of the mean extension of the network strands. The interpretation of equations $(7,9)$ is simple. If a sample is deformed, the equilibrium positions of the junction points change affinely (Eq. (8)). The fluctuations, on the other hand, depend only on the connectivity but not on size and shape of the network. The shear modulus of the phantom model can therefore be calculated without having to integrate out the dynamic eigenmodes of the network.

It is easy to see that dividing the network strands into Gaussian sub-strands (i.e. formally introducing additional, two-functional junction points along the strands) changes nothing for a phantom network. The spring constant is doubled for a strand of half the original length, i.e. if an entropic spring of spring constant $k$ is replaced by a linear sequence of $N$ springs the latter have a spring constant of $N k$. Furthermore, the equilibrium positions of the new cross-links are along the line connecting the equilibrium positions of the original endpoints. Since $\left\langle R_{N}^{2}\right\rangle=$ $\frac{1}{N^{2}}\left\langle R^{2}\right\rangle$, one finds $k N \sum_{i=1}^{N}\left\langle R_{N}^{2}\right\rangle=k\left\langle R^{2}\right\rangle$ and the predicted modulus remains unchanged.

Finite deformations change the free energy density of the phantom model by

$$
\frac{1}{V} \Delta F_{p h}=\frac{1}{2}\left(\lambda^{2}+\frac{2}{\lambda}-3\right) G_{p h}
$$

and give rise to a normal tension $\left\langle\sigma_{T}\right\rangle=$ $\left\langle\sigma_{x x}-\frac{1}{2}\left(\sigma_{y y}+\sigma_{z z}\right)\right\rangle$, where the $\sigma_{\alpha \alpha}$ are the diagonal elements of the microscopic stress tensor $\langle\overleftrightarrow{\sigma}\rangle=\frac{1}{V} \overleftrightarrow{\lambda} \frac{\partial F}{\partial \overleftarrow{\epsilon}^{t}} \overleftrightarrow{\lambda}^{t}$. This can be shown in general in analogy to the virial theorem (see Sect. 4). For the special case of classical rubber elasticity, equation (10), one finds

$$
\left\langle\sigma_{T}\right\rangle(t \rightarrow \infty)=\left(\lambda^{2}-\frac{1}{\lambda}\right) G
$$

by writing the change in the free energy density for a deformation of a sample of size $L_{0}^{3}$ in terms of normal forces $F_{\alpha \alpha}$ acting on the side walls of the sample and expressing the forces via pressures:

$$
\begin{aligned}
L_{0}^{3} d f & =G L_{0}^{3}\left(\lambda-\lambda^{-2}\right) d \lambda=F_{x x} d x+F_{y y} d y+F_{z z} d z \\
& =\left(F_{x x}-\left(F_{y y}+F_{z z}\right) /\left(2 \lambda^{3 / 2}\right)\right) L_{0} d \lambda \\
& =\left(\sigma_{x x} L_{0}^{2} / \lambda-\frac{1}{2}\left(\sigma_{y y}+\sigma_{z z}\right) \lambda^{1 / 2} L_{0}^{2} / \lambda^{3 / 2}\right) L_{0} d \lambda \\
& =\sigma_{T} L_{0}^{3} \lambda^{-1} d \lambda .
\end{aligned}
$$

\section{Fluctuations}

Although the eigenmodes do not contribute to the shear modulus of the phantom model, they have nevertheless received considerable attention $[3,41-43]$. This was due to the problem of how to calculate $\left\langle\mathbf{R}_{i j}{ }^{2}\right\rangle$ for a randomly cross-linked network. Here we re-derive the result from some simple and general considerations.

Consider a randomly cross-linked melt and a melt of the unconnected strands. For instantaneous cross-linking the statistics of the strand conformations and, in particular, $\left\langle r_{i j}^{2}\right\rangle$ is the same in both cases [4]. The precise value follows from applying the equipartition theorem to the melt of unconnected strands: $\frac{k}{2}\left\langle r_{i j}^{2}\right\rangle=\frac{3}{2} k_{B} T$. The internal "energy" of the network, on the other hand, is given by the energy of the equilibrium conformation $E_{0}=\frac{k}{2}\left\langle R_{i j}^{2}\right\rangle$ plus $\frac{1}{2} k_{B} T$ per fluctuating mode. The number of modes equals three times the number of junction points and latter is for an $f$-functional network given by $\frac{2}{f}$ times the number of strands. This corresponds to a thermal energy of $3 \times \frac{2}{f} \times \frac{1}{2} k_{B} T$ per strand, i.e. $\frac{k}{2}\left\langle u_{i j}^{2}\right\rangle=\frac{3}{f} k_{B} T$ and $\frac{k}{2}\left\langle R_{i j}^{2}\right\rangle=\left(\frac{3}{2}-\frac{3}{f}\right) k_{B} T:$

$$
\begin{aligned}
\left\langle u_{i j}^{2}\right\rangle & =\frac{2}{f}\left\langle r^{2}\right\rangle \\
\left\langle R_{i j}^{2}\right\rangle & =\left(1-\frac{2}{f}\right)\left\langle r^{2}\right\rangle .
\end{aligned}
$$

As a result, the shear modulus of the phantom model can be written in a form, where it only depends on the strand density and the functionality of the network [3]:

$$
G_{p h}=\left(1-\frac{2}{f}\right) \rho_{\text {strand }} k_{B} T .
$$

In principle, one can calculate the exact eigenmodes or generalized Rouse modes from the knowledge of the connectivity matrix for a particular network [42,44]. In the appendix we propose an intuitive approximation, where the movements of the junction points and of the strands between them are considered to be independent.

\section{The constrained mode model}

The phantom model completely neglects "entanglements" between network strands due to their mutual impenetrability. The classical view of the problem, associated 
with the name of Flory, is to assume that their main effect is a partial suppression of the junction fluctuations [5-9]. The non-classical theories of rubber elasticity discuss constraints like tubes [11-14] or slip-links [15,21,45] that in addition also restrict the fluctuations of the strands between the junction points.

Often these constraints are introduced as additional, single-node terms into the phantom model Hamiltonian, which constrain the movement of the monomers and junction points. The standard choice are anisotropic, harmonic springs of strength $\overleftrightarrow{l}(\lambda)$ between the nodes and points $\boldsymbol{\xi}_{i}(\lambda)$ which are fixed in space:

$$
\mathcal{H}_{\text {constr }}=\sum_{i} \frac{1}{2}\left(\mathbf{r}_{i}-\boldsymbol{\xi}_{i}(\lambda)\right)^{t} \overleftrightarrow{l}(\lambda)\left(\mathbf{r}_{i}-\boldsymbol{\xi}_{i}(\lambda)\right)
$$

Equivalently one can restrict the nodes to box-like regions of width $\boldsymbol{\delta}(\lambda)=\left(\delta_{x}(\lambda), \delta_{y}(\lambda), \delta_{z}(\lambda)\right)$ :

$$
\begin{aligned}
\exp \left(-\mathcal{H}_{\text {constr }} / k_{B} T\right) & =\prod_{i} \Theta\left(\mathbf{r}_{i}-\left(\boldsymbol{\xi}_{i}(\lambda)\right.\right. \\
- & \left.\left.\frac{1}{2} \boldsymbol{\delta}(\lambda)\right)\right) \Theta\left(\left(\boldsymbol{\xi}_{i}(\lambda)+\frac{1}{2} \boldsymbol{\delta}(\lambda)\right)-\mathbf{r}_{i}\right),
\end{aligned}
$$

where $\Theta(\mathbf{r}) \equiv \Theta(x) \Theta(y) \Theta(z)$ is the product of the step functions of the components of the vector.

Here we study a slightly different constraint model, where instead of restricting the motion of individual nodes, deformation dependent generalized forces couple to the eigenmodes $\mathbf{u}_{p}$ of the phantom network:

$$
\mathcal{H}_{\text {constr }}=\sum_{p} \frac{1}{2}\left(\mathbf{u}_{p}-\mathbf{v}_{p}(\lambda)\right)^{t} \overleftrightarrow{l_{p}}(\lambda)\left(\mathbf{u}_{p}-\mathbf{v}_{p}(\lambda)\right)
$$

The idea to base the analysis on (single chain) eigenmodes is not new, but has apparently not been fully exploited. Edwards and Vilgis [13] proposed a tube model where the primitive path is given by constrained long-wavelength eigen (Rouse) mode of the chain and the fluctuations are due to unimpeded short-wavelength Rouse modes. In the appendix we derive analoguous results in the framework of our "constrained mode model" (CMM). In a similar spirit Duering, Kremer, and Grest $[25,46]$ tried to determine the shear elastic properties of rubber-like materials from the partial relaxation of (approximate) eigenmodes in the undeformed state. Here we provide a more formal base for this ansatz and as a result propose a modified formula for the shear relaxation modulus.

As for the comparison of the confinement generated by the two types of constraining Hamiltonians, equations $(15,17)$, we note that both can be used to model straight tubes with unimpeded fluctuations of the chain parallel to the tube axes. And while both models allow one to arbitrarily displace the average positions of the monomers from their equilibrium positions in the phantom model, both also loose the anisotropic character of the monomer diffusion parallel and perpendicular to a twisted, random walk-like tube. In what they differ is that equation (15) neglects correlations between the restoring forces acting on different monomers (and therefore suppresses also short-wavelength fluctuations), while equation (17) neglects that correlations between different Rouse modes allow to displace the chain along the tube axes without generating restoring forces. However, while neither model is exact, the CMM equation (17) has the big advantage that it can be solved without further approximations.

As the sum of the phantom and constrained mode model Hamiltonians equations $(1,17)$ is diagonal and quadratic in the modes, the model is straightforward to solve. Consider one Cartesian component $\alpha$ of a particular mode $\mathbf{u}_{p}$. Under the influence of the constraining force, the mode has a non-zero mean excitation

$$
U_{p \alpha}(\lambda)=\frac{v_{p \alpha}(\lambda)}{k_{p} / l_{p \alpha \alpha}(\lambda)+1}
$$

and the fluctuations around this mean value are reduced

$$
\left\langle\delta u_{p \alpha}^{2}\right\rangle \equiv\left\langle\left(u_{p \alpha}-U_{p \alpha}(\lambda)\right)^{2}\right\rangle=\frac{k_{B} T}{k_{p}+l_{p \alpha \alpha}(\lambda)} .
$$

In the most general case the deformation dependent free energy has the form

$$
\begin{aligned}
F_{p \alpha}(\lambda)= & \frac{k_{p}}{2} U_{p \alpha}(\lambda)^{2}+\frac{l_{p \alpha \alpha}(\lambda)}{2}\left(v_{p \alpha}(\lambda)-U_{p \alpha}(\lambda)\right)^{2} \\
& +\frac{k_{B} T}{2} \log \left(\frac{k_{p}+l_{p \alpha \alpha}(\lambda)}{k_{B} T}\right),
\end{aligned}
$$

while the contribution of the $p$ th mode to the shear modulus is given by $\frac{1}{3} \frac{d^{2}}{d \lambda^{2}}\left(F_{p x}+F_{p y}+F_{p z}\right)$. The first two terms in equation (20) represent elastic energies stored in the mean excitations of the mode and the constraining spring respectively. In contrast to the phantom model, equation (7), the log-term, representing the fluctuations, is deformation dependent.

The parameters of any constrained fluctuation model have to be chosen in such a way that the average strand conformations in the unstrained state remain unchanged compared to the phantom model. This condition is very easy to express for the constrained mode model. While in the absence of constraints a mode undergoes thermal fluctuations of width $\left\langle u_{p \alpha}^{2}\right\rangle=\frac{k_{B} T}{k_{p}}$, the excitations in the constrained mode model are the sum of the nonvanishing mean excitations equation (18) and the (reduced) thermal excitations equation (19). By demanding that $\left\langle u_{p \alpha}^{2}\right\rangle=\left\langle U_{p \alpha}^{2}\right\rangle+\left\langle\delta u_{p \alpha}^{2}\right\rangle$ one obtains

$$
\begin{aligned}
\left\langle v_{p \alpha}^{2}\right\rangle & =\frac{1}{\gamma_{p}}\left\langle u_{p \alpha}^{2}\right\rangle \\
\left\langle U_{p \alpha}^{2}\right\rangle & =\gamma_{p}\left\langle u_{p \alpha}^{2}\right\rangle \\
\left\langle\delta u_{p \alpha}^{2}\right\rangle & =\left(1-\gamma_{p}\right)\left\langle u_{p \alpha}^{2}\right\rangle \\
\gamma_{p} & =\frac{l_{p}}{k_{p}+l_{p}} .
\end{aligned}
$$

The parameter $\gamma_{p}$ measures how strongly the fluctuations of the $p$ th mode are confined. The extreme cases are $\gamma_{p}=0$ 
and $\gamma_{p}=1$ corresponding to completely free and completely frozen fluctuations respectively. Note, that averaging over the quenched disorder (free energies etc. are calculated with fixed vectors $\mathbf{v}_{p}(\lambda)$ assigned to each mode and averaged afterwards over the (Gaussian) distribution of constraints characterized by $\left.\left\langle v_{p \alpha}^{2}\right\rangle\right)$ does not require any further approximations.

The limiting cases impose certain restrictions on the deformation dependence of the parameters $\overleftrightarrow{l_{p}}(\lambda)$ and $\mathbf{v}_{p}(\lambda)$. The following arguments apply, strictly speaking, only to the constrained mode model equation (17), since previous constrained junction and tube models introduced constraints on the motion of individual monomers, equations $(15,16)$. However as we will show that relations which have been used in order to improve the agreement with experimentally measured stress-strain curves lead to inconsistencies in the present case, one might wonder, if these problems are more general and not just an artifact of the slightly different manner the constraints are introduced in equation (17).

As already noted by Flory, the centers of the constraints have to move affinely with the sample

$$
\mathbf{v}_{p}(\lambda)=\overleftrightarrow{\lambda} \mathbf{v}_{p}
$$

This is, for example, necessary in order to ensure that completely frozen (i.e., in general, long-wavelength) modes with $\gamma_{p}=1$ deform affinely with the sample. In the limit of $\gamma_{p}=0$ equation (20) can be rewritten as $F_{p \alpha}\left(\lambda, \gamma_{p}=0\right)=\frac{K_{B} T}{2} \frac{l_{p \alpha \alpha}(\lambda)}{l_{p \alpha \alpha}}\left(\frac{v_{p \alpha}(\lambda)}{v_{p \alpha}}\right)^{2}$. Since unconstrained fluctuations must not contribute to the shear modulus, the deformation dependence of $v_{p \alpha}(\lambda)$ and $l_{p \alpha}(\lambda)$ may not be chosen independently. The simplest choice consistent with this requirement is:

$$
\overleftrightarrow{l_{p}}(\lambda)=(\overleftrightarrow{\lambda})^{-2} \overleftrightarrow{l_{p}}
$$

which can be visualized as cavities that move and deform affinely with the sample. In particular, it is not possible to let the strength of the constraints vary with a different power as in some tube models [14]. Flory and Erman proposed more complicated relations of the type $l_{p} / l_{p}(\lambda)=\lambda^{2}(1+\gamma \zeta(\lambda-1))$ in our notation. By varying the parameter $\zeta$ they were able to improve the agreement of their constrained junction model with measured stress-strain curves. However, at least in the present case, this ansatz leads to inconsistencies. Demanding that $\lim _{\gamma \rightarrow 1} G=1$, limits one's freedom in choosing to $\zeta=0$ or $\zeta=1$, and in the remaining non-trivial case one finds $\left.\frac{d}{d \gamma} G\right|_{\gamma=0}<0$ or negative contributions of weakly constrained modes to the shear modulus. Nevertheless, there seem to be other possibilities. At least, we have not found any theoretical arguments that would exclude, for example, $l_{p} / l_{p}(\lambda)=\lambda^{2}\left(1+\gamma^{2}(\lambda-1)\right)$. As a consequence, equation (22) involves an assumption and we will later come back to the point how it may be tested in computer simulations.

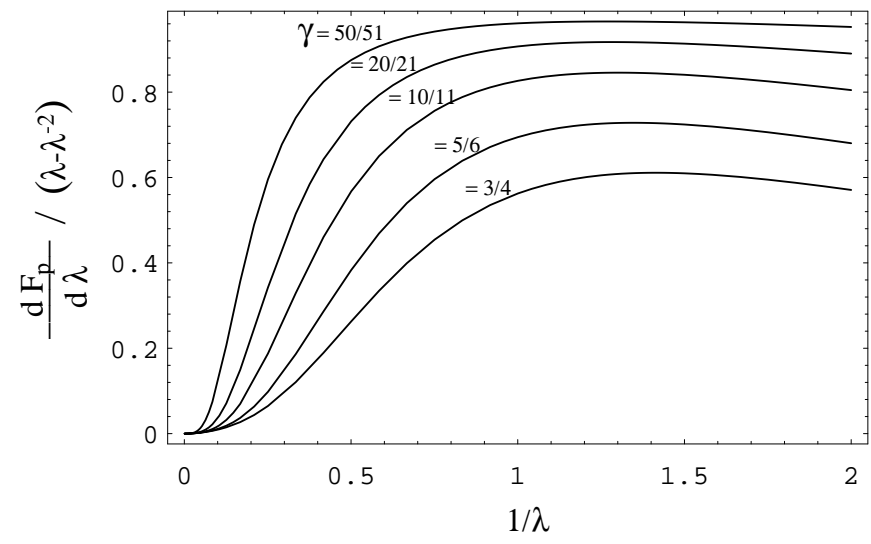

Fig. 1. Contribution of a partially constrained mode to the restoring forces of a deformed network in Mooney-Rivlin representation. The values of $\gamma=\frac{\kappa}{\kappa+1}$ were chosen to correspond to Figure 2 in Flory's first paper on constrained junction models [6].

Some general conclusions can be drawn with respect to the microscopic deformations in the constrained mode model with equations $(21,22)$. The mean-excitations,

$$
\frac{U_{p \alpha}(\lambda)}{U_{p \alpha}(1)}=\frac{\lambda_{\alpha \alpha}}{(1-\gamma) \lambda_{\alpha \alpha}^{2}+\gamma},
$$

of partially frozen modes deform $s u b$-affinely. Only in the limit $\gamma \rightarrow 1$ one finds $U_{p}(\lambda)=\lambda U_{p}(1)$. A similar result holds for the width of the fluctuations:

$$
\frac{\left\langle\delta u_{p \alpha}^{2}(\lambda)\right\rangle}{\left\langle\delta u_{p \alpha}^{2}(1)\right\rangle}=\frac{\lambda_{\alpha \alpha}^{2}}{(1-\gamma) \lambda_{\alpha \alpha}^{2}+\gamma} .
$$

While the fluctuations are deformation independent for unconstrained modes, their width increases sub-affinely for $0<\gamma<1$. For completely frozen modes with $\gamma \equiv$ $1,\left\langle\delta u_{p \alpha}^{2}(\lambda)\right\rangle \equiv 0$ independent of $\lambda$. Finally, one obtains for the deformation dependent free energy and the shear modulus of the constrained mode model:

$$
\begin{aligned}
F_{p \alpha}(\lambda)= & \frac{K_{B} T}{2} \frac{1}{1+\gamma_{p}\left(\lambda_{\alpha \alpha}^{-2}-1\right)} \\
& +\frac{k_{B} T}{2} \log \left(1+\frac{\gamma_{p}}{1-\gamma_{p}} \lambda_{\alpha \alpha}^{-2}\right) \\
G_{c m}= & G_{p h}+\frac{k_{B} T}{V} \sum_{p} \gamma_{p}^{2} .
\end{aligned}
$$

Inspection of equation (25) shows that one recovers the ideal stress-strain behavior equation (11) only in the two limiting cases of $\gamma=0$ and $\gamma=1$. In Figure 1 we have plotted some results for intermediate values in the MooneyRivlin form often used to represent experimental data. Qualitatively, the curves are very similar to those obtained from Flory's constrained junction model [6] and we can therefore expect the same, good agreement with experimental data. However, as we have made no particular assumptions about which modes are constrained by the 
entanglements, it is clear that nothing is to be learned from the shape of the stress-strain curves about the validity of the classical models of rubber elasticity.

\section{Discussion}

While the constrained mode model as presented above is formally very simple, an obvious difficulty is hidden in the employment of the eigenmodes of the phantom model. In principle, it is possible to calculate these so-called generalized Rouse modes from the knowledge of the connectivity matrix for a particular network $[42,44]$. In the appendix we work out an intuitive approximation which in a similar form is employed by all constrained fluctuation models of rubber elasticity, where the movements of the individual junction points and network strands are considered to be independent. For the fluctuations of the junction points around their mean positions we follow Flory and use an Einstein-model. For the network strands we follow the community insofar as we employ single chain Rouse modes, but insist on the apparently uncommon choice of using boundary conditions which correspond to fixed as opposed to free chain ends. Such Rouse modes can be used to describe the fluctuations of monomers around their respective equilibrium positions. Together the Einstein and the Rouse modes form a complete and orthogonal basis set. In particular, they are like the true eigenmodes independent of size and shape of the network. In contrast to the Rouse modes for long paths through the network, which were used by Duering et al. $[25,46]$ to analyze their simulation data, the autocorrelation functions of the present modes decay to zero for non-entangled systems. In fact, the two types of modes allow a much clearer distinction between classical and non-classical entanglement effects. In the appendix we show how (i) by constraining the Einstein modes one obtains the crossover from the phantom to the affine model described by the constrained junction models of classical rubber elasticity [5-9] and how (ii) by additionally constraining the Rouse modes one recovers the tube model [11-14] with a strand length independent shear modulus. In the general case, the CMM allows one to study the whole crossover from networks with strand lenghts $N \ll N_{e}$, which are well-described by classical rubber elasticity, to entanglement dominated systems with $N \gg N_{e}$.

Even with the simplified mode spectrum, the CMM is probably still too detailed (i.e., has too many free parameters $\gamma_{p}$ ) to be tested quantitatively in experiments. As we have already pointed out, the worst approach is probably to rely on a fit of rheological data to the stress-strain curves that follow from equation (25) regarding $\gamma$ as a parameter characterizing the average confinement of whatever modes. Judging from the inconclusiveness of previous investigations along these lines [31-37], it is clear that more microscopic input is needed. But even neutron scattering experiments $[29,30,47]$ are limited to comparing the data to structure factors calculated from models. Clearly, more information on, for example, the wave-length dependence of the parameters $\gamma_{p}$ of the constrained mode model is needed, before a fit could be attempted. We note that recent data are in very good agreement with a variant of the tube model which tries to account for non-affine deformations of both, the tube axes and diameter $[29,30]$. As we have pointed out, such effects appear rather naturally in the framework of the constrained mode model as a consequence of partially frozen modes with $0<\gamma_{p}<1$.

While the great number of parameters $\gamma_{p}$ of the constrained mode model is disadvantageous for a comparison with experiments, the opposite is true for the interpretation of simulation data. Current molecular dynamics simulations of model polymer networks cover the relevant time and length scales and allow to directly determine the restoring forces in strained systems $[38,39,48-50]$. At the same time, and in contrast to experiments, one has access to the actual chain conformations. This allows one to measure the parameters $\gamma_{p}$ of the CMM in a simulation of an undeformed network. In subsequent runs of the same system under strain one can directly test the predictions of the CMM for the microscopic deformations, equations $(23,24)$, and the elastic properties, i.e., for the deformation dependence of the network free energy equation (25), the corresponding restoring forces, the small strain shear modulus equation (26), and the modified mode analysis expression (B.1) for the shear relaxation modulus $G(t)$ $[25,46]$.

As a last point we address a general aspect of all constrained fluctuation models of rubber elasticity, namely the relation between the polymer contribution to the stress tensor and the deformation dependence of the constraints. Within the CMM, equation (25), the normal tension $\sigma_{T}=\frac{\lambda}{V} \frac{d F}{d \lambda}$ induced by a deformation equation (5) can be written in the form

$$
\begin{aligned}
\left\langle\sigma_{T}\right\rangle= & \frac{1}{V} \sum_{\langle i, j<i\rangle} k\left(X_{i j}^{2}(\lambda)-\frac{1}{2}\left(Y_{i j}^{2}(\lambda)+Z_{i j}^{2}(\lambda)\right)\right) \\
& +\frac{1}{V} \sum_{p} k_{p}\left\langle u_{p x}^{2}(\lambda)-\frac{1}{2}\left(u_{p y}^{2}(\lambda)+u_{p z}^{2}(\lambda)\right)\right\rangle .
\end{aligned}
$$

One the one hand, one might well have expected this result. Equation (27) is the equivalent of the Doi and Edwards [11] formula for the contribution of free polymers to the stress tensor and has the same form as the virial theorem (see below) for harmonic springs. On the other hand, it is quite surprising that the CMM should yield the same (measurable!) expression as the phantom model without constraints, i.e. that the constraints in the CMM do not contribute directly to the restoring forces, even though equation (20) shows that they store elastic energy.

To better understand this apparent contradiction we re-derive in the following the virial theorem for deformation dependent interactions. It turns out that the validity of the Doi-Edwards stress formulas is bound to nontrivial conditions on the deformation dependence of the constraints. In fact, one recovers equation (27) for any theoretical model that augments the phantom model 
Hamiltonian by terms which become deformation invariant under affine transformations $\mathbf{r} \rightarrow \overleftrightarrow{\lambda} \mathbf{r}$.

Consider a general Hamiltonian $\mathcal{H}=\sum_{i<j} U\left(\left|\mathbf{r}_{i j}\right|\right)$ (e.g. the phantom model Hamiltonian Eq. (1)) without constraints. Using scaled co-ordinates $\mathbf{r}_{i}=\overleftrightarrow{\lambda} \mathbf{s}_{i}$, distances are calculated by contraction with the metric tensor $\overleftrightarrow{\Lambda}=\overleftrightarrow{\lambda}^{t} \overleftrightarrow{\lambda}$. By this change of variables the deformation dependence of the partition function is transferred from the integration bounds to the Hamiltonian:

$$
\begin{aligned}
& \mathcal{Z}=\operatorname{det}(\overleftrightarrow{\lambda})^{N} \\
& \times \int d s^{3 N} \exp \left[-\frac{1}{k_{B} T} \sum_{i<j} U\left(\sqrt{\mathbf{s}_{i j}^{t} \overleftrightarrow{\Lambda} \boldsymbol{s}_{i j}}\right)\right] .
\end{aligned}
$$

Differentiation of the free energy $F=-k_{B} T \log (\mathcal{Z})$ with respect to the deformation parameter $\lambda$ shows that systems responds to a deformation equation (5) by developing a normal tension:

$$
\begin{aligned}
\frac{1}{V} \frac{d F}{d \lambda} & =\frac{1}{V}\left\langle\frac{d \mathcal{H}}{d \lambda}\right\rangle \\
& =\frac{1}{\lambda}\left\langle\sigma_{x x}-\frac{1}{2}\left(\sigma_{y y}+\sigma_{z z}\right)\right\rangle=\frac{\sigma_{T}}{\lambda}
\end{aligned}
$$

where

$$
\begin{aligned}
\sigma_{\alpha \beta} & =\frac{2}{V} \overleftrightarrow{\lambda} \frac{d \mathcal{H}}{d \Lambda_{\alpha \beta}} \overleftrightarrow{\lambda} t \\
& =\frac{1}{V} \sum_{i<j} \frac{U^{\prime}\left(r_{i j}\right)}{r_{i j}}\left(\mathbf{r}_{i j}\right)_{\alpha}\left(\mathbf{r}_{i j}\right)_{\beta}
\end{aligned}
$$

is the instantaneous stress tensor. For harmonic interactions, as in the phantom and Rouse model Hamiltonians for polymer melts and networks, equation (30) reduces to the Doi and Edwards [11] formula for the polymer contribution to the stress tensor. Differentiating twice and taking the limit $\lambda \rightarrow 1$ yields an expression for the small strain elastic modulus:

$$
\begin{aligned}
3 G= & \left\langle\operatorname{Tr} \overleftrightarrow{\sigma^{\prime}}\right\rangle-\frac{V}{k_{B} T}\left(\left\langle\sigma_{T}^{2}\right\rangle-\left\langle\sigma_{T}\right\rangle^{2}\right) \\
& +\frac{1}{V}\left\langle\sum_{i<j}\left(U^{\prime \prime}-\frac{U^{\prime}}{r_{i j}}\right) \frac{\left(x_{i j}^{2}-\frac{1}{2}\left(y_{i j}^{2}+z_{i j}^{2}\right)\right)^{2}}{r_{i j}^{2}}\right\rangle .
\end{aligned}
$$

Similar expression for other types of deformation have been discussed in the literature and are widely used in simulations $[49,51,52]$. For the phantom and Rouse model Hamiltonians the last, so-called hyper-virial term vanishes. In these cases equation (31) describes the polymer contribution to the shear modulus in analogy to the Doi and Edwards [11] formula for the stress tensor.

Now consider the constraint-Hamiltonian equation (17). If one uses the scaled variables, it becomes independent of the deformation provided the constraints deform affinely as in equations $(21,22)$. The same holds for the constraint-Hamiltonians equations $(15,16)$. In such a case, differentiating the free energy with respect to $\lambda$ yields the same expressions as for the phantom model without constraints. The expectation values do, of course, change as the averages are taken over a different ensemble. For theories which employ non-affinely deforming constraints additional terms appear, for example, in the stress-tensor equation (30). This general result is interesting, since in a computer simulation equations $(30,31)$ can be evaluated for the true microscopic as well as for the effective entropic interactions ${ }^{2}$. A comparison of equations $(26,31)$ for the shear modulus, on the other hand, provides a direct test for the importance of correlations between different modes.

To summarize, with the constrained mode model (CMM) equation (17) we have introduced an exactly solvable constrained fluctuation model of rubber elasticity which is particularly suited for the analysis of simulation data. It differs from most other models in that deformation dependent linear forces couple to the eigenmodes of the phantom network instead of restricting the motion of individual monomers or junction points. Nevertheless, the tube model as well as the constrained junction models of classical rubber elasticity can be recovered as limiting cases in an approximation where the true eigenmodes are replaced by a combination of Einstein and Rouse modes for the movements of the junction points and network strands respectively. In the general case, the CMM allows one to study the whole crossover from networks with strand lengths $N \ll N_{e}$ which are well-described by classical rubber elasticity to entanglement dominated systems with $N \gg N_{e}$. In the CMM the confinement of the motion of each mode is characterized by a single parameter $\gamma$ and sub-affine microscopic deformations, equations $(23,24)$, and deviations from the ideal stress-strain behavior (Fig. 1) are predicted as a result of partial confinement of modes with $0<\gamma<1$. The small strain shear modulus of the CMM, equation (26), has a particularly simple form and suggests a modification of the mode analysis expression equation (B.1) for the shear relaxation modulus $G(t)$ proposed by Duering et al. [25,46]. Quite surprisingly, we find that the CMM can only be formulated consistently for affinely deforming constraints, equations $(21,22)$. While this is, of course, no formal proof that the same restrictions also apply to other constrained fluctuation models, we have shown in analogy to the derivation of the virial theorem that for a system with constraints the usual DoiEdwards formulas for the polymer contribution to the stress tensor also require affinely deforming constraints. In this case, one is in the interesting situation that the constraints only contribute explicitely to the deformation free energy but not to the tensions in a strained system.

\footnotetext{
${ }^{2}$ Such a comparison has already been carried out for an entangled melt, but not for a cross-linked melt with permanent topological constraints. Gao and Weiner [53] showed that the Doi-Edwards formula does work in the reptation regime, i.e. on the time-scales where there is no fundamental difference between a melt and a network.
} 
I gratefully acknowledge intense discussions with K. Kremer and G.S. Grest on how we should analyze our simulation data. Without their input, their criticism and their patience this work would not have been possible. I have also benefitted from discussions with J. Baschnagel, B. Dünweg, M. Gottlieb, J.-F. Joanny, A. Johner, E. Straube, W. Wintz, and H.-P. Wittmann.

\section{Note added}

After this work was finished Rubinstein and Panyukov [54] published a paper where they develop a complementary view of the relation between non-affine chain deformations and corrections to the neo-hookian elasticity. These results are obtained using a constraint-Hamiltonian of the type of equation (15) for virtual chains coupled to the backbone of long network strands and effectively confining them to tube-like regions. Note that as in the CMM the strength of the confining potential is assumed to vary affinely with the deformation.

\section{Appendix A: Classical and tube models as limiting cases of the constrained mode model}

In principle, one can calculate the exact eigenmodes or generalized Rouse modes from the knowledge of the connectivity matrix for a particular network $[42,44]$. Here we propose an approximation, where the movements of the junction points and of the strands between them are considered to be independent. The ansatz has the advantage that it provides an intuitive distinction between the classical and tube contributions to the shear modulus.

\section{A.1. Constraining the motion of the junction points}

Classical rubber elasticity only considers the extension of the network strands as a whole and can therefore be formulated in terms of the motion of the junction points. The following approximation corresponds to the Einsteinmodel in solid-state physics and was, for example, also used by Flory to estimate the contribution of the deformation dependent fluctuations to the shear modulus of his constrained junction model.

Consider a particular junction point $i$ of an $f$ functional network. If one assumes that its topological neighbors are fixed at their equilibrium positions $\mathbf{R}_{j}$, a displacement $u_{i \alpha}$ of node $i$ in one spatial direction requires an energy $(f k / 2) u_{i \alpha}^{2}$. Treating these displacements as independent eigenmodes ("Einstein modes") with spring constant $k_{p}=f k$, one can use the equipartition theorem to obtain $\left\langle u_{i \alpha}^{2}\right\rangle=\frac{k_{B} T}{f k}$. As a consistency check, we note that this result implies that the extension of a particular network strand should undergo thermal fluctuations of a width $\left\langle\left|\mathbf{u}_{i j}\right|^{2}\right\rangle=2\left\langle\left|\mathbf{u}_{i}\right|^{2}\right\rangle=2 \times 3 \frac{k_{B} T}{f k}=\frac{2}{f}\left\langle r^{2}\right\rangle$ in agreement with equation (12).

To demonstrate that constraining these modes has the same effects as predicted by the conventional constrained junction models [5-9] we show that the shear modulus calculated from the constrained mode model interpolates between the phantom and junction affine network limits. That partially constrained modes lead to the typical Mooney-Rivlin deviations from the ideal stress-strain relation equation (11) was already discussed in Section 3.

While it is obvious from equation (26) that $G_{c m}$ reduces to $G_{p h}$ for $\gamma_{p} \equiv 0$, one needs to know the actual number of modes to calculate $G_{c m}$ in the opposite limit of $\gamma_{p} \equiv 1$. Using our result from Section 2 that there are $2 / f$ (three component) modes per network strand and equation (14) for the shear modulus of the phantom model one finds

$$
\begin{aligned}
G_{c m}(\gamma \equiv 1) & =G_{p h}+\frac{2}{f} \rho_{\text {strand }} k_{B} T \\
& =\rho_{\text {strand }} k_{B} T \\
& =G_{\text {aff }} .
\end{aligned}
$$

The junction affine model [4] is, in fact, the oldest model of rubber elasticity. The assumption, that the surrounding molecules suppress the movements of the junction points so strongly that the latters' instantaneous positions (and not only their mean positions as in the case of the phantom model) change affinely with the shape of the sample, leads immediately to the above results for $G_{a f f}$.

In reference [48] we quantitatively tested the classical theories in computer simulations of model polymer networks. We determined the true shear moduli by measuring the restoring forces in deformed networks and calculated parameter-free predictions from the phantom and the junction affine model. By showing that the measured shear moduli exceeded $G_{a f f}$, we provided the first quantitative proof that the classical theories overlook important contributions to the entropy change in a deformed network.

However, in the light of the present results our analysis of the constrained junction models appears incorrect. In reference [48] we calculated the classical modulus from the observed deformation dependence of single strand entropies. In the context of the constrained mode model this corresponds to analyzing quantities of the type

$$
\begin{aligned}
E(\lambda) & =\frac{k_{p}}{2}\left\langle\mathbf{r}_{i j}{ }^{2}(\lambda)\right\rangle \\
& =\frac{k_{p}}{2}\left(\left\langle\mathbf{R}_{i j}{ }^{2}(\lambda)\right\rangle+2\left\langle U_{p \alpha}^{2}(\lambda)\right\rangle+2\left\langle\delta u_{p \alpha}^{2}(\lambda)\right\rangle\right)
\end{aligned}
$$

which are identical to the proper free energies equation (20) only in the limits $\gamma=0$ and $\gamma=1$. While evaluating the free energy of the constrained mode model is quite cumbersome (the constraining springs are not directly observable!), we show in Section 4 that one can elegantly circumvent this problem by comparing measured and calculated normal tensions.

\section{A.2. Constraining the strand fluctuations between the junction points}

The non-classical theories of rubber elasticity discuss constraints like tubes, slip-links $[15,45]$ or the presence of filler 
material, that restrict the fluctuations of the strands between the junction points. Treating the strands as independent, these fluctuations are most naturally analyzed in terms of single chain Rouse-modes [11]. Consider a linear Gaussian chain of $N_{\text {strand }}+1$ beads with a Hamiltonian $\mathcal{H}=\frac{k N_{\text {strand }}}{2} \sum_{i=1}^{N_{\text {strand }}}\left(\mathbf{r}_{i}(t)-\mathbf{r}_{i-1}(t)\right)^{2}$ and the spring constants $k N_{\text {strand }}$ chosen as discussed in Section 2 . For the present purposes, it turns out to be convenient to regard the end points as fixed at $\mathbf{r}_{0}(t) \equiv \mathbf{R}_{0}$ and $\mathbf{r}_{N_{\text {strand }}}(t) \equiv \mathbf{R}_{N_{\text {strand }}}$, instead of the usual open boundary conditions. One can then write the the deviations $\mathbf{u}_{i}(t)=\mathbf{r}_{i}(t)-\mathbf{R}_{i}$ from the equilibrium positions $\mathbf{R}_{i}=\mathbf{R}_{0}+i / N_{\text {strand }}\left(\mathbf{R}_{N_{\text {strand }}}-\mathbf{R}_{0}\right)$ in terms of sinRouse-modes:

$$
\begin{aligned}
\mathbf{u}_{p}(t) & =\frac{1}{N_{\text {strand }}+1} \sum_{i=0}^{N_{\text {strand }}} \mathbf{u}_{i}(t) \sin \left(\frac{p \pi i}{N_{\text {strand }}}\right) \\
\mathcal{H} & =\frac{k}{2}\left(\mathbf{R}_{0}-\mathbf{R}_{N_{\text {strand }}}\right)^{2}+\sum_{p} \frac{k_{p}}{2} \mathbf{u}_{p}{ }^{2} \\
k_{p} & =\frac{2 \pi^{2} k}{N_{\text {strand }}} p^{2} .
\end{aligned}
$$

As the Einstein modes describe the fluctuations of the junction points, the Rouse modes equation (A.1) describe the fluctuations of the monomers around their respective equilibrium positions. Together the Einstein and the Rouse modes form a complete and orthogonal basis set. In particular, they are like the true eigenmodes independent of size and shape of the network and allow an intuitive distinction between classical and non-classical contributions to the shear modulus.

The correspondence with the tube model is most conveniently discussed in the limit of long strands of $N_{\text {strand }} \rightarrow \infty$ Gaussian units, where the classical contribution to the shear modulus becomes negligible: $G_{\text {class }} \leq$ $G_{a f f}=\frac{\rho}{N_{\text {strand }}} k_{B} T \rightarrow 0$ ( $\rho$ here denotes the number density of the Gaussian units). Consider, for example, the ansatz

$$
\gamma_{p}=\left\{\begin{array}{l}
1 \text { for } p<\frac{N_{\text {strand }}}{N_{e}} \\
0 \text { for } p>\frac{N_{\text {strand }}}{N_{e}}
\end{array}\right.
$$

which freezes all modes with a wavelength larger than a certain "entanglement length" $N_{e}$. The meansquare distance between the equilibrium positions of neighboring Gaussian units, $\left\langle\left(\mathbf{U}_{i}-\mathbf{U}_{i+1}\right)^{2}\right\rangle=$ $8 \sum_{p} \gamma_{p} \frac{k_{B} T}{k_{p}} \sin ^{2}\left(\frac{p \pi}{N_{\text {strand }}}\right)$, as calculated from the first $N_{\text {strand }} / N_{e}$ frozen modes is of the order of $\left\langle r^{2}\left(N_{e}\right)\right\rangle / N_{e}^{2}$. The corresponding tube axes has a length of $\frac{N_{\text {strand }}}{N_{e}} \sqrt{\left\langle r^{2}\left(N_{e}\right)\right\rangle}$ and deforms affinely with the sample. The tube width, on the other hand, is given by the fluctuations of a monomer around its equilibrium position, $\left\langle\delta \mathbf{u}_{i}^{2}\right\rangle=2 \sum_{p}\left(1-\gamma_{p}\right) \frac{k_{B} T}{k_{p}}$, and of the order of $R_{g}^{2}\left(N_{e}\right)$ in- dependently of the deformation. Finally, the shear modulus is obtained by multiplying the number of frozen modes per chain, $N_{\text {strand }} / N_{e}$, with the chain density $\rho / N_{\text {strand }}$ :

$$
G_{\text {tube }}=\frac{N_{\text {strand }}}{N_{e}} \frac{\rho}{N_{\text {strand }}} k_{B} T=\frac{\rho}{N_{e}} k_{B} T .
$$

As already discussed in Section 3, partially frozen modes with $0<\gamma_{p}<1$ lead to a weaker than affine deformations of both, the tube axes and the tube diameter, and to the characteristic Mooney-Rivlin corrections to the ideal stress-strain curves.

\section{Appendix B: A mode analysis expression for the shear relaxation modulus}

In this section we comment on the mode analysis as it was used by Duering et al. $[25,46]$ to estimate the shear relaxation function of model polymer networks in computer simulations of undeformed samples. A heuristic generalization of the shear modulus equation (26) of the constrained mode model for finite times is

$$
G_{c m}(t)=G_{p h}+\frac{k_{B} T}{V} \sum_{p}\left(\frac{\left\langle\mathbf{u}_{p}(t) \cdot \mathbf{u}_{p}(0)\right\rangle}{\left\langle\mathbf{u}_{p}^{2}\right\rangle}\right)^{2}
$$

where the sum over the modes includes both, the Einstein and the Rouse modes (A.1) for the fluctuations of the junctions points and the monomers around their respective equilibrium positions. Equation (B.1) has the correct limiting behavior for large times and reduces to the Rousemodel result [11]

$$
G_{\text {Rouse }}(t)=G_{p h}+\frac{k_{B} T}{V} \sum_{p} e^{-2 t / \tau_{p}}
$$

for $\gamma=0$. The factor of two in the exponential between the auto-correlation function of a mode $\left\langle\mathbf{u}_{p}(t) \cdot \mathbf{u}_{p}(0)\right\rangle=$ $\left\langle\mathbf{u}_{p}{ }^{2}\right\rangle \exp \left(-t / \tau_{p}\right)$ and its contribution to the shear relaxation modulus lead Duering et al. $[25,46]$ to the slightly different ansatz

$$
G(t)=\frac{k_{B} T}{V} \sum_{p} \frac{\left\langle\mathbf{X}_{p}(2 t) \cdot \mathbf{X}_{p}(0)\right\rangle}{\left\langle\mathbf{X}_{p}^{2}\right\rangle} \stackrel{t \rightarrow \infty}{\longrightarrow} \frac{k_{B} T}{V} \sum_{p} \gamma_{p}
$$

where the $\mathbf{X}_{p}$ are now the usual free chain Rouse modes

$$
\begin{aligned}
\mathbf{X}_{p}(t)= & \frac{1}{N_{\text {path }}} \sum_{i=1}^{N} \mathbf{r}_{i}(t) \cos \left(\frac{p \pi(i-1)}{N_{\text {path }}-1}\right) \\
& -\frac{1}{2 N_{\text {path }}}\left[\mathbf{r}_{1}(t)+(-1)^{p} \mathbf{r}_{N_{\text {path }}}(t)\right]
\end{aligned}
$$

used for the analysis of the conformations of long paths through the network. Equation (B.3) does not agree with equation (26), but reduces by construction to the Rouse result equation (B.2). 
Why do we now propose to use the network strand Rouse modes, $\mathbf{u}_{p}$, equation (A.1), instead of the free chain Rouse modes, $\mathbf{X}_{p}$, equation (B.4)? First of all, we note that even for a phantom network the auto-correlation functions of the free chain Rouse modes, $\left\langle\mathbf{X}_{p}(t) \cdot \mathbf{X}_{p}(0)\right\rangle$, do not decay to zero. This is simply due to the fact that each network strand has in contrast to a free chain a nonvanishing mean extension. As a consequence, the signature of permanent entanglements in polymer networks is much less clear, when the conformations are analyzed in terms of the free chain Rouse modes, than when they are analyzed in terms of the network strand Rouse modes. There the incomplete decay of a mode auto-correlation function $\left\langle\mathbf{u}_{p}(t) \cdot \mathbf{u}_{p}(0)\right\rangle$ directly signals the existence of effects which are ignored by the classical theories of rubber elasticity. Also, since the free chain Rouse modes when applied to a phantom network become deformation dependent, they are only of limited use for the analysis of the conformations of strained networks. While these are practical reasons for the use of the network strand Rouse modes, equation (A.1), the difference between equation (B.1) and equation (B.3) suggests that the question of which type of modes is used is not just a matter of convenience. According to the results we obtained for the constrained mode model there is a subtle difference in how the equilibrium extensions of the network strands contribute to the shear modulus. The part which is due to the cross-linking and treated by the phantom model contributes linearly (Eq. (14)), while the part that is due to the constraining of fluctuations, $\gamma=\frac{\left\langle U_{p \alpha}^{2}\right\rangle}{\left\langle u_{p \alpha}^{2}\right\rangle}$, contributes only quadratically (Eq. (26)). Using the free chain Rouse modes this difference is lost and the modulus over-estimated. In order to make the distinction and to use equation (26), it is necessary to employ modes which as the true eigenmodes of the phantom model depend only on the connectivity of the network.

\section{References}

1. H. James, J. Chem. Phys. 15, 651 (1947).

2. H. James, E. Guth, J. Chem. Phys. 15, 669 (1947).

3. P.J. Flory, Proc. Royal Soc. Lond. A. 351, 351 (1976).

4. L.R.G. Treloar, The Physics of Rubber Elasticity (Clarendon Press, Oxford, 1975).

5. G. Ronca, G. Allegra, J. Chem. Phys. 63, 4990 (1975).

6. P.J. Flory, J. Chem. Phys. 66, 5720 (1976).

7. B. Erman, P.J. Flory, J. Chem. Phys. 68, 5363 (1978).

8. P.J. Flory, B. Erman, Macromol. 15, 800 (1982).

9. S. Kästner, Colloid Polym. Sci. 259, 499 and 508 (1981).

10. S.F. Edwards, Proc. Phys. Soc. 92, 9 (1967).

11. M. Doi, S.F. Edwards, The Theory of Polymer Dynamics (Claredon Press, Oxford, 1986).

12. W.W. Graessley, Adv. Pol. Sci. 47, 67 (1982).

13. S.F. Edwards, T.A. Vilgis, Rep. Progr. Phys. 51, 243 (1988).
14. G. Heinrich, E. Straube, G. Helmis, Adv. Pol. Sci. 85, 34 (1988).

15. R.C. Ball, M. Doi, S.F. Edwards, M. Warner, Polymer 22, 1010 (1981).

16. G. Marrucci, Macromol. 14, 434 (1981).

17. R.J. Gaylord, J. Poly. Bull. 8, 325 (1982).

18. S.F. Edwards, T.A. Vilgis, Polymer 27, 483 (1986).

19. D. Adolf, Macromol. 21, 228 (1988).

20. M. Kosc, Colloid Polym. Sci. 266, 105 (1988).

21. P.G. Higgs, R.C. Ball, Europhys. Lett. 8, 357 (1989).

22. K. Kremer, G.S. Grest, J. Chem. Phys. 92, 5057 (1990).

23. W. Paul, K. Binder, D.W. Heermann, K. Kremer, J. Phys. II France 1, 37 (1990).

24. W. Paul, K. Binder, D.W. Heermann, K. Kremer, J. Chem. Phys. 95, 7726 (1991).

25. E.R. Duering, K. Kremer, G.S. Grest, J. Chem. Phys. 101, 8169 (1994).

26. D. Richter et al., Phys. Rev. Lett. 64, 1389 (1990).

27. D. Richter et al., Macromol. 25, 6156 (1992).

28. D. Richter et al., Phys. Rev. Lett. 71, 4158 (1993).

29. E. Straube, V. Urban, W. Pyckhout-Hintzen, D. Richter, Macromol. 27, 7681 (1994).

30. E. Straube et al., Phys. Rev. Lett. 74, 4464 (1995).

31. M. Gottlieb et al., Macromol. 14, 1039 (1981).

32. M. Gottlieb, R.J. Gaylord, Polymer 24, 1644 (1983).

33. M. Gottlieb, R.J. Gaylord, Macromol. 17, 2024 (1984).

34. M. Gottlieb, R.J. Gaylord, Macromol. 20, 130 (1987).

35. B. Erman, P.J. Flory, Macromol. 15, 806 (1982).

36. S.K. Patel, S. Malone, C. Cohen, J.R. Gillmor, Macromol. 25, 5241 (1992).

37. M.A. Sharaf, J.E. Mark, Polymer 35, 740 (1994).

38. R. Everaers, K. Kremer, G.S. Grest, Macromol. Symposia 93, 53 (1995).

39. K. Kremer, G.S. Grest, in Monte-Carlo and Molecular Dynamics Simulations in Polymer Science, edited by K. Binder (Oxford University Press, New York and Oxford, 1995).

40. R. Everaers, K. Kremer, in preparation; G.S. Grest, R. Everaers, K. Kremer, in preparation.

41. J.A. Duiser, A.J. Stavermann, in Physics of noncrystalline solids, edited by J. Prins (North-Holland, Amsterdam, 1965), p. 376.

42. B.E. Eichinger, Macromol. 5, 496 (1972).

43. W.W. Graessley, Macromol. 8, 186 and 865 (1975).

44. J.U. Sommer, M. Schulz, H.L. Trautenberg, J. Chem. Phys. 98, 7515 (1993).

45. W.W. Graessley, D.S. Pearson, J. Chem. Phys. 66, 3363 (1977).

46. E.R. Duering, K. Kremer, G.S. Grest, Phys. Rev. Lett. 67, 3531 (1991).

47. T.A. Vilgis, F. Boué, Polymer 27, 1154 (1986).

48. R. Everaers, K. Kremer, Macromol. 28, 7291 (1995).

49. S.J. Barsky, M. Plischke, Phys. Rev. E 53, 871 (1996).

50. S.J. Barsky, M. Plischke, Z. Zhou, B. Joós, Phys. Rev. E 54, 5370 (1996).

51. J.R. Ray, Comp. Phys. Rep. 8, 109 (1988).

52. Z. Zhou, B. Joós, Phys. Rev. B 54, 3841 (1996).

53. J. Gao, J.H. Weiner, J. Chem. Phys. 103, 1614 (1995).

54. M. Rubinstein, S. Panyukov, Macromol. 30, 8036 (1997). 